\title{
Heart Rate Response to Dypiridamole Stress in Relation to Perfusion and Function during Gated Technetium Tc99 M Sestamibi Spect Study
}

\section{Abstract}

Objectives: A mild decrease in blood pressure and increase in heart rate (HR) are considered normal hemodynamic responses to dipyridamole. In this study, we tried to investigate the relation between the responses of heart rate to dipyridamole stress test with left ventricular perfusion and function data obtained from myocardial perfusion scintigraphy.

Methods: Forty consecutive patients undergoing dipyridamole stress Technetium - 99m (Tc 99m) Sesta MIBI gated myocardial perfusion single photon emission computed tomography (SPECT) at the nuclear cardiology Lab of the cardiology department of the main university hospital of Alexandria University were prospectively enrolled from July 2013 for 6 months. Dipyridamole was infused over $4 \mathrm{~min}$ and TC $99 \mathrm{~m}$ Sesta MIBI was injected 2 min after the end of infusion. Blunted heart rate response to dipyridamole considered if the HR ratio (peak HR/rest HR) was 1.20 or less. Summed stress score (SSS), summed rest score (SRS), and summed difference score (SDS) for myocardial perfusion in addition to total perfusion defect TPD, ejection fraction and territory of perfusion defect were obtained.Patients were grouped according to HR response and groups were compared. A logistic regression analysis was used to determine independent predictors of reduced HR response.

Results: Blunted heart rate response was found in $67.5 \%$ of patients. Patients with abnormal heart rate response were more frequently had a history of diabetes mellitus $\left(X^{2}=4.01\right)$. High baseline heart rate $(t=1.9)$, low post stress ejection fraction $(t=3.21)$ and perfusion defect in left circumflex territory $\left(\mathrm{X}^{2}=\right.$ 4.01) was found in blunted heart rate response group more than normal group.

Conclusion: Severe perfusion defectis associated with blunted heart rate group as left ventricular dysfunction is associated with reduced chronotropic response to dipyridamole.Diabetes mellitus and cardiac autonomic neuropathy may reflect higher baseline heart rate.

Keywords: Myocardial perfusion scintigraphy; Heart rate; Diabetes mellitus; Left ventricular failure; Autonomic dysfunction

\author{
Research Article \\ Volume 2 Issue 3 - 2015 \\ Ahmed Abdel Aaty, AmrThanaa El- \\ DeenZaky, Mohammed Ibrahim Lotfy and \\ Ahmed Mohammed Fathy* \\ Department of Cardiology, Alexandria University, Egypt \\ *Corresponding author: Ahmed Mohammed Fathy, \\ Department of Cardiology, Faculty of Medicine, \\ Alexandria University, Egypt, Tel: 201115200150; Email: \\ cardioegypt@ymail.com
}

Received: January 22, 2015 | Published: April 01, 2015
Abbreviations: SSS: Summed Stress Score; SRS: Summed Rest Score; SDS: Summed Difference Score; CAD: Coronary Artery Disease; LVEF: Leftventricular Ejection Fraction

\section{Introduction}

The heart rate of an individual reflects an integrated physiological response comprising of autonomic system, central and peripheral reflexes as well as intrinsic cardiac conditions. An abnormal heart rate response to physiological stress may be secondary to multiple metabolic abnormalities such as diabetes or renal failure as well as intrinsic cardiac conditions such as coronary artery disease (CAD) and cardiomyopathy [1]. Subsequently, Ellestad et al. [2] demonstrated that a slowheart rate response during exercise was a greater predictor of cardiac events than stress - induced ischemic ST depression and coined the term "chronotropic incompetence" for these responses. Dipyridamole is an indirect coronary artery vasodilator which results in a mild increase in heart rate (HR) and a mild decrease in both systolic and diastolic blood pressures [3]. Lee et al. [4] demonstrated blunted heart rate response to dipyridamole in diabetic patients and explained this phenomenon by autonomic neuropathy.

The increased risk of death found in patients witha blunted HR response to dipyridamole, even in the presence ofnormal perfusion, reinforces the importance of the search for thereasons for this phenomenon [5]. Therefore,the aim of this study was to investigate theassociation demographic, hemodynamic and gated SPECT variables and severity of perfusion defect, ejection fraction and if the blunted heart rate is related to specific territory in patients undergoing myocardial perfusion scintigraphy.

\section{Materials and Methods}

\section{Study population}

Adult patients undergoing stress/rest dipyridamole Tc99m sesta MIBI SPECT study 2 days protocol in the Nuclear Cardiology Lab. Cardiology department in Alexandria Main 
University Hospital from July 2013 for 6 months, 40 patients were enrolled in the study (27 male, 13 female, mean age $58 \pm$ 7.33, range 42-73) after signature of an informed consent. All of them answered a questionnaire with clinical information. Hypertension was defined by history of BP > 140/90 mm Hg and/orantihypertensive drug use; diabetes was defined by history of diagnosis made by a physician and/or use of insulin or oral hypoglycemic medications. Exclusion criteria were the presence of tachy arrhythmias, second or third degree AV block, pacemaker rhythm, congestive heart failure (NYHA classes III/ IV) or significant valvular heart disease.

\section{Dipyridamole protocol}

Patients were instructed not to smoke nor consume caffeinated food and beverages nor methyl-xanthene's containing products for 12 hours before testing and to With hold beta-blockers, calcium channel blockers and nitrates for at least 24 - $48 \mathrm{~h}$ prior to the study. Dipyridamole is administered at 0.56 $\mathrm{mg} / \mathrm{kg}$ intravenously over a 4-min period. A 12 lead ECG was monitored continuously. HR and blood pressure were measured at rest and every min after the initiation of dipyridamole infusion for a total period of 6 min. Tc-99m sesta MIBI was injected at 6 minutes after onset of infusion. The occurrence of symptoms was asked at the time of and after the infusion of dipyridamole. Aminophylline was intravenously given 2 minutes after radiotracer injection at peak stress in a dose of 120 to $240 \mathrm{mg}$ to reverse any adverse effect caused by dipyridamole. Significant ST segment depression during dipyridamole stress was defined as $\geq 1 \mathrm{~mm}$ of horizontal or down sloping depression occurring at 80 milli seconds after the J point. Peak HR and BP values were defined as the highest $\mathrm{HR}$ and lowest $\mathrm{BP}$ during the observation period (before the administration of aminophylline, if that happened), since the effects of dipyridamole may be still relevant after the completion of the infusion. We calculated the HR ratio (HR at peak/HR at rest), the difference between peak and rest HR (delta HR) and peak and rest systolic BP (delta BP). An attenuated HR response to dipyridamole was considered present if the HR ratio was $\leq 1.20$, a value derived from the study of Bhateja et al. [5].

\section{SPECT protocol}

2 day stress/ rest protocol was performed by Tc-99m sestaMIBI myocardial perfusion SPECT in all patients. For stress imaging, Tc-99m sestaMIBI (15-30 mCi) was injected intravenously after 2 minutes of finishing pharmacological stress (with dipyridamole). Eight-framegated MPS imaging acquisition was started 60 minutes after radioisotope injection. Resting image in the second day was performed after 60 minutes of injection of Tc- $99 \mathrm{~m}$ sestaMIBI (15-30 mCi). Image acquisitions were obtained using Siemens Symbia E Gamma Camera with ECG gating.

\section{Image interpretation}

Semi quantitative visual interpretation of MPS images was performed with short axis and vertical long axis tomograms divided into 17 segments. Each segment was scored using a 5 -point scale $(0=$ normal; $1=$ equivocal; $2=$ moderate; $3=$ severe reduction of tracer uptake; $4=$ absence of detectable radiotracer activity in a segment). The summed stress score (SSS), a measure of the total perfusion defect, and the summed restscore (SRS), a measure of the rest defect meaning infarcted tissue, were obtained by means of adding the scores for the 17 segments ofthe stress and rest images, respectively. The difference between the summed stress score and SRS was defined as the summed difference score (SDS), a measure of the amount of myocardial ischemia. After automatic reorientation, post stress gated shortaxis images were processed using quantitative gated SPECT software and left ventricular ejection fraction (LVEF), enddiastolic volume, and end-systolic volume were automatically calculated.

\section{Case study (group A)}

Sixty five years old male patient hypertensive, not diabetic and non-smoker. The patient complaint of atypical chest pain with no dyspnea. During dipyridamole stress, his resting HR is 58 beat /minute whilepeak HR is 71 beat / minute. HR ratio > 1.2 , his study revealed mild ischemia in RCA territory. There was transient ischemic dilatation during stress. TPD during stress $=3 \%$ and during rest $2 \%$. EF during stress and rest $77 \%$. ECG revealed right bundle branch block with no changes after stress (Figure 1)

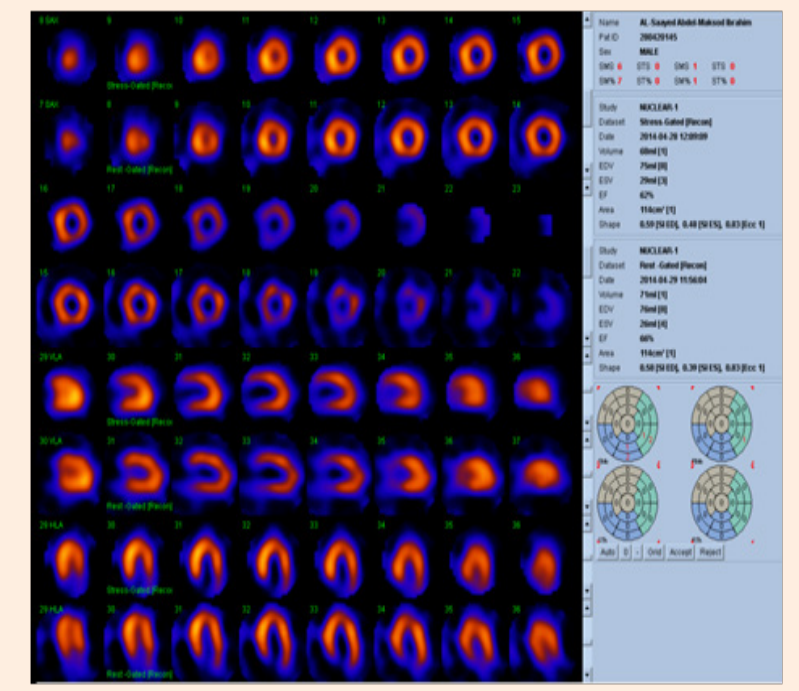

Figure 1: EF during stress and rest $77 \%$.

\section{Case study (group B)}

Fifty six years old male patient hypertensive, diabetic, ischemic and smoker. During dipyridamole stress testing, his resting HR was 82 beat/ minute while peak HR was 85 beat/ minute, $\mathrm{HR}$ ratio $<1.2$, the study revealed an extensive perfusion defect involving apex, septum, anteroseptal, inferoseptal and inferior wall from the apex up to the base. There was apical scar and aneurysm. LV was permanent dilated during stress and rest. The delayed resting with reinjection revealed partial reperfusion in the septum, anteroseptal, inferoseptal and inferior wall. TPD during stress $41 \%$ and during rest $30 \%$. EF during stress $31 \%$ and during rest $34 \%$ (Figure 2). 


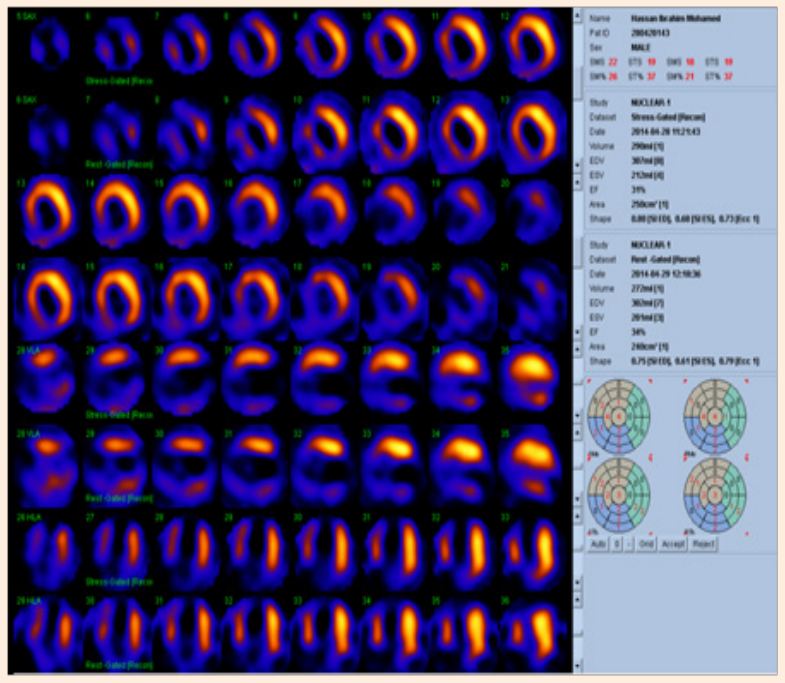

Figure 2: EF during stress $31 \%$ and during rest $34 \%$.

\section{Results}

Study population had high prevalence of hypertension and Diabetes mellitus (70 and 37\% respectively). Demographic data of the patients are shown in Table 1.13 patients $(32.5 \%)$ had normal HR response and 27 (67.5\%) patients have reduced HR response to dipyridamole. There was no significant difference by means of gender, age, history of IHD and smoking. Hypertension predominates in reduced heart rate group $(77.7 \%)$ to normal heart rate group $(53.8 \%)$ but with no statistical significance. The prevalence of diabetes mellitus was significantly high in reduced HR response group [48 vs. $15.3 \%\left(\mathrm{p}=0.04^{*}\right)$ ]. By studying the hemodynamics (Table 2), resting HR was higher in blunted HRR group than normal HRR group with statistically significant value $(\mathrm{P}=0.03)$. Resting and peak blood pressure had not a significant difference between 2 groups. Perfusion study (Table 3) demonstrate a significant perfusion defect noticed in blunted HRR group as well as post stress EF was highly different between 2 groups and dropping of EF was noticed in blunted HRR group ( $48.9 \pm 10.7$ Vs. $62.5 \pm 9.2)$ to normal HRR group. Blunted HRR group is associated with a perfusion defect in LCX and LAD territory.

Table 1: Demographic data, risk factors, clinical presentation and ECG changes of total population, normal and reduced HRR groups.

\begin{tabular}{|c|c|c|c|c|c|}
\hline & $\begin{array}{c}\text { Total population } \\
\qquad n=40\end{array}$ & $\begin{array}{c}\text { Normal HRR } \\
n=13\end{array}$ & $\begin{array}{c}\text { Reduced HRR } \\
n=27\end{array}$ & Test of sig. & $\mathbf{P}$ \\
\hline Age (year) & $58 \pm 7.33$ & $58.4 \pm 6.9$ & $58 \pm 8.23$ & $\mathrm{t}=0.1$ & $\mathrm{p}=0.9$ \\
\hline \multicolumn{6}{|c|}{ Sex } \\
\hline Male & $25(62.5 \%)$ & $10(76.9 \%)$ & 15 (55.5\%) & $X^{2}=1.7$ & $\mathrm{P}=0.191$ \\
\hline Female & $15(37.5 \%)$ & $3(23 \%)$ & $12(44.5 \%)$ & & \\
\hline \multicolumn{6}{|c|}{ Risk Factors } \\
\hline $\mathrm{Dm}$ & 15 (37.5\%) & $2(15.3 \%)$ & $13(48.1 \%)$ & $X^{2}=4.01$ & $\mathrm{P}=0.04^{*}$ \\
\hline Hypertension & $28(70 \%)$ & $7(53.8 \%)$ & $21(77.7 \%)$ & $\mathrm{X}^{2}=1.55$ & $\mathrm{P}=0.212$ \\
\hline F.H of IHD & $10(25 \%)$ & $2(15.3 \%)$ & $8(29.6 \%)$ & $X^{2}=0.94$ & $\mathrm{P}=0.32$ \\
\hline Smoking & $11(27.5 \%)$ & $3(23 \%)$ & $8(29.6 \%)$ & $X^{2}=0.18$ & $\mathrm{P}=0.66$ \\
\hline \multicolumn{6}{|c|}{ Symptoms } \\
\hline Chest Pain & $36(90 \%)$ & $13(100 \%)$ & $23(85.1 \%)$ & $X^{2}=2.13$ & $\mathrm{P}=0.143$ \\
\hline Dyspnea & $19(47.5 \%)$ & $5(38.4 \%)$ & $14(51.8 \%)$ & $X^{2}=0.63$ & $\mathrm{P}=0.427$ \\
\hline
\end{tabular}

Table 2: Hemodynamics parameters in normal HRR and RHRR groups.

\begin{tabular}{|c|c|c|c|c|c|}
\hline & Total pop. & Normal HRR & Reduced HRR & Test of sig. & P value \\
\hline \multicolumn{7}{|c|}{ Heart Rate } \\
\hline Resting & $75.7 \pm 14.8$ & $69.4 \pm 13.4$ & $78.7 \pm 14.7$ & $\mathrm{t}=1.9$ & $\mathrm{p}=0.03^{*}$ \\
\hline Peak & $84.3 \pm 13.8$ & $88.3 \pm 14$ & $82.41 \pm 13.55$ & $\mathrm{t}=1.27$ & $\mathrm{p}=0.2$ \\
\hline Delta HR & $8.65 \pm 8.81$ & $18.9 \pm 5.6$ & $3.7 \pm 4.85$ & $\mathrm{t}=8.8$ & $\mathrm{P}<0.001^{*}$ \\
\hline \multicolumn{7}{|c|}{ Blood Pressure } & & $\mathrm{p}$ \\
\hline Resting Syst. & $153 \pm 32.2$ & $146.1 \pm 33.3$ & $157.4 \pm 31.6$ & $\mathrm{t}=1.03$ & $\mathrm{p}=0.3$ \\
\hline Peak Syst. & $140.75 \pm 21.9$ & $134.6 \pm 25.3$ & $143.7 \pm 19.8$ & $\mathrm{t}=1.23$ & $\mathrm{p}=0.22$ \\
\hline
\end{tabular}


Table 3: SPECT parameters in normal HRR and reduced HRR groups.

\begin{tabular}{|c|c|c|c|c|c|}
\hline & Total pop. & Normal HRR & Reduced HRR & Test of sig. & $P$ value \\
\hline \multicolumn{6}{|c|}{ Perfuison Study } \\
\hline SSS & $8.55 \pm 8.8$ & $3.3 \pm 2.9$ & $11.07 \pm 9.66$ & $\mathrm{t}=2.7$ & $\mathrm{p}=0.005^{*}$ \\
\hline SRS & $3.6 \pm 6$ & $1.23 \pm 1.4$ & $4.81 \pm 7.09$ & $t=1.77$ & $\mathrm{p}=0.04^{*}$ \\
\hline SDS & $4.72 \pm 4.4$ & $2.07 \pm 2.5$ & $6 \pm 4.69$ & $\mathrm{t}=2.84$ & $\mathrm{p}=0.003^{*}$ \\
\hline TPD & $10.12 \pm 11.58$ & $6.38 \pm 9.6$ & $11.92 \pm 12.15$ & $\mathrm{t}=2.3$ & $\mathrm{p}=0.02^{*}$ \\
\hline \multicolumn{6}{|c|}{ Severity } \\
\hline 1)normal & $9(22.5 \%)$ & $4(30.7 \%)$ & $5(18.5 \%)$ & \multirow{4}{*}{$Z=-2.02$} & \multirow{4}{*}{$\mathrm{P}<0.04 *$} \\
\hline 2) mild & $14(35 \%)$ & 7 (53.8\%) & $7(25.9 \%)$ & & \\
\hline 3)moderate & $10(25 \%)$ & $2(15.3 \%)$ & $8(29.9 \%)$ & & \\
\hline 4) sever & 7 (17.5\%) & $0(0.0 \%)$ & $7(25.9 \%)$ & & \\
\hline \multicolumn{6}{|c|}{ Distribution } \\
\hline 1)LAD & $18(45 \%)$ & $4(30.7 \%)$ & $14(51.85 \%)$ & $X^{2}=1.57$ & $\mathrm{P}=0.2$ \\
\hline 2) RCA & $13(32.5 \%)$ & $6(46.1 \%)$ & $7(25.9 \%)$ & $X^{2}=1.63$ & $\mathrm{P}=0.2$ \\
\hline 3)LCX & $16(40 \%)$ & $3(23 \%)$ & $13(48.1 \%)$ & $X^{2}=4.01$ & $\mathrm{P}=0.04^{*}$ \\
\hline 4)2VESS & $9(22.5 \%)$ & $4(30.7 \%)$ & $5(18.5 \%)$ & $X^{2}=0.11$ & $\mathrm{P}=0.7$ \\
\hline 5)3VESS & $5(12.5 \%)$ & $0(0.0 \%)$ & $5(18.5 \%)$ & $X^{2}=2.7$ & $\mathrm{P}=0.09$ \\
\hline \multicolumn{6}{|c|}{ LV study } \\
\hline post stress EF & $52.5 \pm 11.44$ & $62.5 \pm 9.2$ & $48.9 \pm 10.7$ & $t=3.21$ & $\mathrm{p}=0.002^{*}$ \\
\hline LV size & $11(27.5 \%)$ & $3(23 \%)$ & $8(29.6 \%)$ & $\mathrm{X}^{2}=0.18$ & $\mathrm{P}=0.66$ \\
\hline
\end{tabular}

\section{Discussion}

Despite the link between attenuation of the HR response todipyridamole and risk of death [5], similar to the increased mortality found in patients with chronotropic incompetence during exercise [6], the pathophysiology of this phenomenon is not completely understood. Lee et al. [4] have ascribed a low HR response to dipyridamole in diabetic patients to cardiac autonomic neuropathy; however, their findings were obtained in selected patients with normal perfusion images and no history of coronary artery disease, ascenario which neither mirrors the routine of the myocardial perfusion imaging laboratory - the evaluation of patients with known or suspected coronary artery disease - nor mirrors the actual diabetic population, whose wellknown high prevalence of coronary artery disease may lead to abnormal MPS images.

In our study, blunted HR response to dipyridamole was frequent $(67.5 \%)$ and associated with $\mathrm{LV}$ dysfunction as found with Kim et al. [7] who described a correlation between reduced chronotropic response to dipyridamole and LVEF in post-myocardial infarction patients. Infact, chronotropic incompetence is not uncommon in left ventricular dysfunction [8]. Increased serum catecholamine levels and increased sympathetic tone during heart failure may be responsible for blunted effects of vasodilators on heart rate [9]. High rest HR might be considered not only a sign of cardiacautonomic neuropathy [10], but also of left ventricular dysfunction [11]. The most significant Pvalue found for LVEF may suggest that left ventricular dysfunction is the strongest predictor of blunted ofthe HR response to dipyridamole.

Recent evidence indicates that inflammation and sustained tachycardia interact at several levels of the cardiovascular continuum [12], and may here by exert a synergistic effect on cardiovascular morbidity and mortality. For example, elevated heart rate increases tensile stress which apart from inducing endothelial injury also increases endothelial permeability to circulating inflammatory mediators [13]. Dysfunctional autonomic nervousactivity may underlie both progressions of inflammation as well as elevated resting heart rate [14]. Elevated sympathetic activity modifies the inflammatory processand thereby promotes endothelial dysfunction and subsequent at hero progression [15]. So, The European Society of Hypertension/European Society of Cardiology guidelines recently proposed the inclusion of elevated heart rate when evaluating the cardiovascular risk profile of an individual [16].

Parameters of perfusion defect like SSS, SRS, SDS and TPD had significant value in our study with blunted HR group that found increasing the severity, the more prominent blunted HR $\left(\mathrm{P}<0.05^{*}\right)$. Andrea De Lorenzo et al. [17] recommended in his study that finding of blunted HR response is strongly associated with reduced $\mathrm{EF}$ and elevated resting $\mathrm{HR}$. 
By studying the coronary artery lesions provided by coronary angiogram and its perfusion defect by MPI we found that significant lesions in LCX and LAD territories that supply left ventricle were associated with blunted heart rate response. This proves that any factor affect TPD and LV dysfunction is associated with blunted response to dipyridamole. By studying RCA (that is supplying SAN) lesions, we found $25 \%$ only of blunted HRR to dipyridamole had RCA territory perfusion defect. In another word, SAN affection is not a risk factor of blunted HRR to dipyridamole, and this blunting HRR is more physiological attributed to LV dysfunction than anatomical (SAN affection). So the limitation of our study include; inflammatory markers was not investigated that may explain of presence or absence of blunted HR in some cases.

\section{Conclusion}

Blunted HR response to dipyridamoleis independent predictor of the severity of perfusion defect and reduced post stress EF. Perfusion defect in LCX territory was prominent in blunted HR response group than other territories. Elevated resting $\mathrm{HR}$ is associated with increasing morbidity and mortality.

\section{References}

1. Hinkle LE, Carver ST, Plakun A (1972) Slow heart rates and increased risk of cardiac death in middle-aged men. Arch Intern Med 129(5): 732-748.

2. Ellestad MH, Wan MK (1975) Predictive implications of stress testing: Follow-up of 2700 subjects after maximum treadmill stress testing. Circulation 51(2): 363-369.

3. Iskandrian AS, Verani MS, Heo J (1994) Pharmacologic stress testing: mechanism of action, hemodynamic responses, and results in detection of coronary artery disease. J Nucl Cardiol 1(1): 94-111.

4. Lee K, Yoon JK, Lee MG (2001) Dipyridamole myocardial SPECT with low heart rate response indicates cardiac autonomic dysfunction in patients with diabetes. J Nucl Cardiol 8(2): 129-135.

5. Bhateja R, Francis GS, Pothier CE (2005) Heart rate response during dipyridamole stress as a predictor of mortality in patients with normal myocardial perfusion and normal electrocardiograms. Am J Cardiol 95(10): 1159-1164.

6. Elhendy A, Mahoney DW, Khandheria BK (2003) Prognostic significance of impairment of heart rate response to exercise: impact of left ventricular function and myocardial ischemia. J Am Coll Cardiol 42(5): 823-830.

7. Kim YH, Lee KH, Chang HJ, Lee EJ, Chung HW, et al. (2006) Depressed heart rate response to vasodilator stress for myocardial SPECT predicts mortality in patients after myocardial infarction. Int J Cardiovasc Imaging 22(5): 663-670.

8. Clark AL, Coats AJ (1995) Chronotropic incompetence in chronic heart failure. Int J Cardiol 49(3): 225-231.

9. Dhalla AK, Wong MY, Wang WQ, Biaggioni I, Belardinelli L (2006) Tachycardia caused by A2A adenosine receptor agonists is mediated by direct sympathoexcitation in awake rats. J Pharmacol Exp Ther 316(2): 695-702.

10. Boulton AJM, Vinik AI, Arezzo JC, Bril V, Feldman EL, et al. (2005) Diabetic neuropathies: a statement by the American Diabetes Association. Diabetes Care 28(4): 956-962.

11. Esler M, Kaye D, Lambert G, Esler D, Jennings G (1997) Adrenergic nervous system in heart failure. Am J Cardio 80(11A): 7L-14L.

12. Zamorano JL (2008) Heart rate management: a therapeutic goal throughout the cardiovascular continuum. European Heart Journal Supplements 10(Suppl F): F17-F21.

13. Giannoglou GD, Chatzizisis YS, Zamboulis C, Parcharidis GE, Mikhailidis DP, et al. (2008) Elevated heart rate and atherosclerosis: an overview of the pathogenetic mechanisms. Int J Cardiol 126(3): 302-312.

14. Tracey KJ (2002) The inflammatory reflex. Nature 420(6917): 853859.

15. Redwine LS, Wirtz PH, Hong S, Bosch JA, Ziegler MG, et al. (2010) Depression as a potential modulator of Beta-adrenergic-associated leukocyte mobilization in heart failure patients. J Am Coll Cardiol 56(21): 1720-1721.

16. Mancia G, De Backer G, Dominiczak A, Cifkova R, Fagard R, et al (2007) 2007 ESH-ESC Practice Guidelines for the Management of Arterial Hypertension: ESH-ESC Task Force on the Management of Arterial Hypertension. J Hypertens 25(9): 1751-1762.

17. De Lorenzo A, Lima RS (2009) Reduced Heart Rate Response to Dipyridamole as a Marker of Left Ventricular Dysfunction in Diabetic Patients Undergoing Myocardial Perfusion Scintigraphy. Clin Nucl Med 34(5): 275-278 